\title{
Infundibulo-hypophysitis-like radiological image in a patient with pituitary infiltration of a diffuse large B-cell non-Hodgkin lymphoma
}

\author{
A León-Suárez, P Roldán-Sarmiento, M A Gómez-Sámano, A Nava-De la Vega, \\ V M Enríquez-Estrada, F J Gómez-Pérez and D Cuevas-Ramos \\ Department of Endocrinology and Metabolism, Neuroendocrinology Clinic, Instituto Nacional de \\ Ciencias Médicas y Nutrición Salvador Zubirán, Tlalpan, Mexico
}

\author{
Correspondence \\ should be addressed \\ to D Cuevas-Ramos \\ Email \\ ceptamim@gmail.com
}

\section{Summary}

Non-Hodgkin lymphoma (NHL) is a hematological tumor caused by abnormal lymphoid proliferation. NHL can arise in any part of the body, including central nervous system (CNS). However, pituitary involvement is a quite rare presentation. The diffuse large B-cell lymphoma (DLBCL) is the most common subtype when pituitary is infiltrated. Here, we report a case of pituitary infiltration of NHL DLBCL type in a woman with hypopituitarism and an infundibulum-hypophysitis-like image on magnetic resonance imaging (MRI). A female aged 64 years, complained of dyspepsia, fatigue, weight loss and urine volume increment with thirst. Endoscopy and gastric biopsy confirmed diffuse large B-cell lymphoma. Treatment with chemotherapy using R-CHOP was initiated. During her hospitalization, hypotension and polyuria were confirmed. Hormonal evaluation was compatible with central diabetes insipidus and hypopituitarism. Simple T1 sequence of MRI showed thickening of the infundibular stalk with homogeneous enhancement. After lumbar puncture analysis, CNS infiltration was confirmed showing positive atypical lymphocytes. Pituitary and infundibular stalk size normalized after R-CHOP chemotherapy treatment. In conclusion, pituitary infiltration of NHL with infundibular-hypophysitis-like image on MRI is a rare finding. Clinical picture included hypopituitarism and central diabetes insipidus. Diagnosis should be suspected after biochemical analysis and MRI results. Treatment consists of chemotherapy against NHL and hormonal replacement for pituitary dysfunction.

\section{Learning points:}

- Pituitary infiltration by lymphoma can present with signs and symptoms of panhypopituitarism and diabetes insipidus.

- MRI findings can resemble an autoimmune hypophysitis.

- Patients can recover pituitary function as well as normalization of MRI after chemotherapy treatment.

\section{Background}

Diffuse large B-cell lymphoma (DLBCL) is a non-Hodgkin lymphoma (NHL) subtype that commonly cause fever, weight loss and intense sweating or, the well-known, B symptoms. Additional clinical manifestations are related with tumor localization (1). Small proportion of cases have been reported with SNC infiltration $(1,2)$.
Pituitary involvement is particularly rare. Infiltration of DLBCL can cause mass effect symptoms such as headache, visual impairment, diplopia and ocular nerve palsy. Different grades of hormone deficiencies have been reported, including posterior pituitary involvement and central diabetes insipidus (3). However, involvement 
of both pituitary lobes has only been reported in 4 of 9 cases $(3,4)$.

\section{Case presentation}

Here we report a 64-year-old woman previously diagnosed with degenerative osteoarthritis in both knees and type 2 diabetes mellitus at two-year diagnosis. She was under metformin treatment.

She was worried because of dyspepsia, nausea, vomiting, increase in thirst and urine volume with $>3 \mathrm{~L}$ per day. Also she noticed fatigue and weight loss, but no night sweats. Endoscopy showed multiple nodules and ulcers in gastric body, which required several biopsies. Histopathology analysis described diffuse large B-cell lymphoma (CD20+) infiltration, negative for $H$. pylori infection. CT scan was performed and Ann Arbor stage IVB was documented with positive abdominal lymph nodes. Bone marrow analysis was negative for infiltration. R-CHOP (rituximab, cyclophosphamide, vincristine, doxorubicin and prednisolone) chemotherapy was administered.

After 2 weeks of chemotherapy administration, she was hospitalized with an episode of bacteremia. On admission, the patient presented hypotension, hyponatremia and polyuria. Treatment with IV meropenem and norepinephrine was initiated with good response. On physical examination, the patient had dry mouth and skin, without orthostatic hypotension. The remaining clinical evaluation was unremarkable. During her hospitalization, polydipsia and polyuria syndrome persisted, and urine output was quantified between 4 and $5.800 \mathrm{~L}$, urine osmolality between 35 and 70 mosmol (normal: 300-900 mosmol) and serum sodium between 141 and $153 \mathrm{mEq} / \mathrm{L}$ (normal: 135-145 mEq/L).

\section{Investigation}

A dehydration test was performed, with initial serum sodium of $142 \mathrm{mEq} / \mathrm{L}$ and calculated urinary density of 35 mosmol. Serum sodium increases to $153 \mathrm{mEq} / \mathrm{L}$ with persistent polyuria and weight reduction of more than $3 \%$. Then, $0.1 \mathrm{mg}$ desmopressin IV was administered and elevation of urinary density up to 490 mosmol with sodium normalization was documented and central diabetes insipidus was diagnosed.

Pituitary function was evaluated and results confirmed central hypothyroidism with normal TSH $2.99 \mu \mathrm{U} / \mathrm{mL}(0.4-4.5 \mu \mathrm{U} / \mathrm{mL})$, but low free thyroxine

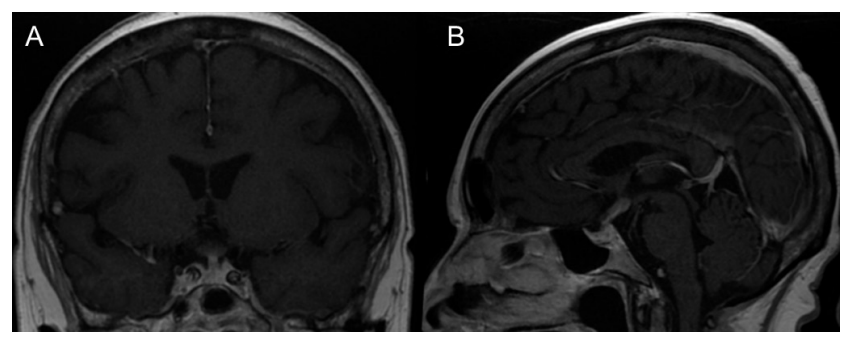

Figure 1

A (coronal) and B (sagittal) contrasted T1 sequences with uniform pituitary gland enhancement and important pituitary stalk thickening, particularly at superior third of the infundibulum (arrows).

level in $0.54 \mathrm{ng} / \mathrm{dL}$ (normal: $0.63-1.34 \mathrm{ng} / \mathrm{dL}$ ); central adrenal insufficiency with basal cortisol serum level of $4.4 \mu \mathrm{g} / \mathrm{dL}$ with maximum cortisol release of $14 \mu \mathrm{g} / \mathrm{dL}$ (expected higher of $18 \mu \mathrm{g} / \mathrm{dL}$ ) after $1 \mu \mathrm{g}$ synthetic adrenocorticotropin (cosyntropin) stimulation test. Also, hypogonadotropic hypogonadism (FSH: $1.34 \mathrm{IU} / \mathrm{mL}$, LH: $0.21 \mathrm{IU} / \mathrm{mL}$ with low estradiol $(18.5 \mathrm{pg} / \mathrm{mL}))$ was documented. Serum prolactin was moderate high $(77 \mathrm{ng} / \mathrm{mL}$, normal: 3-22 ng/mL), with normal age- and genderadjusted IGF-1 level. Contrasted MRI was performed showing pituitary homogeneous enhancement in $\mathrm{T} 1$ sequence with thickening of infundibular stem $(3.9 \mathrm{~mm})$. Optic chiasm and hypothalamus infiltration were also reported (Fig. 1). Lumbar puncture was performed and at least 5 atypical lymphocytes were found, flow cytometry was not performed to the fluid. CNS infiltration diagnosis was supported by the clinical and radiological findings.

\section{Treatment}

Treatment with levothyroxine $100 \mu \mathrm{g} / \mathrm{day}$, oral hydrocortisone $20 \mathrm{mg} /$ day and inhaled desmopressin $(0.1 \mathrm{mg}$ every $12 \mathrm{~h})$ was initiated, with progressive clinical improvement and good resolution of symptoms. The patient received chemotherapy with dexamethasone and methotrexate plus additional systemic IV R-CHOP.

\section{Outcome and follow-up}

After one month, reduction of pituitary stalk thickening was observed on MRI (Fig. 2). The patient received 6 cycles of R-CHOP with 5 high doses of intrathecal methotrexate. The plan was to evaluate the whole pituitary function after the treatment completion, 7 months later, but unfortunately, the patient passed away due to refractory septic shock before that. 


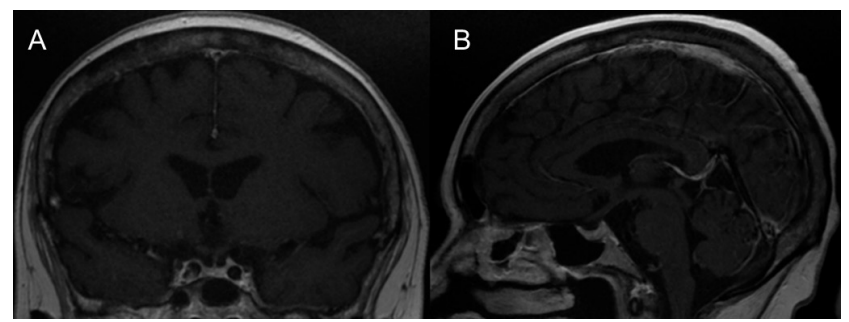

Figure 2

A (coronal) and B (sagittal) contrasted T1 sequences showed uniform pituitary gland enhancement and important reduction in its size. Also, MRI confirmed no further enhancement and thickening of the infundibular stalk after chemotherapy treatment.

\section{Discussion}

Here, we report an interesting case of women affected by a DLBCL subtype with pituitary gland and infundibulum involvement. This is a rare entity with poor clinical experience worldwide; therefore, we believe this case is illustrative and contributes to scientific literature in the field to better understand this type of rare manifestation.

Although DLBCL may involve CNS, pituitary gland is a rare site of infiltration. Anterior and posterior pituitary lobes involvement, showing both panhypopituitarism and central diabetes insipidus is a quite rare presentation. The symptoms that were present in our patient were therefore a combination of hypothyroidism, adrenal insufficiency and diabetes insipidus. Also, dyspepsia was initially reported by the patient because of infiltration of gastric mucosa. However, pituitary infiltration was suspected after she was hospitalized, and hypotension, polyuria and polydipsia were documented. Another additional rare manifestation is anasarca due to hypothyroidism (1).

The most common tumors in the sellar region are adenomas (85-95\%), but the differential diagnoses include inflammatory infiltrative diseases, benign neoplasms and metastatic disease. In the context of a primary tumor, the diagnosis of metastatic disease is the first option (5). Pituitary metastasis accounts for only $1 \%$ of the pituitary tumors (6), the most common are lung cancer (30-50\%) and breast cancer (20-30\%) (7). Of these malignant diseases, systemic lymphoma represents 5-29\%, primarily NHL (8).

Interestingly, our patient showed involvement of both pituitary lobes, which is a more uncommon presentation in patients with pituitary infiltration of lymphoma. The hormonal evaluation revealed hypopituitarism and central diabetes insipidus. The differential diagnoses include autoimmune hypophysitis, infections and granulomatous infiltration. Lymphoma infiltration was not initially suspected because it rarely includes anterior lobe involvement. Usually, presentation is with only central diabetes insipidus (3). Patients diagnosed with lymphoma undergo treatment with corticosteroids at high doses. In some studies, it has been demonstrated that it causes low adrenal response to stress and decrease TSH serum levels. However, after few days of corticosteroids suspension, pituitary hormones return to normal levels (9).

The images obtained with the MRI were much alike for a patient with autoimmune hypophysitis with pituitary homogeneous enhancement, loss of intensity of the posterior pituitary lobe and thickening of the infundibular stalk (10). NHL infiltration of posterior pituitary gland also causes loss of the posterior hyper-intensity on MRI. However, majority of cases also reported a heterogeneous mass infiltration of the adenohypophysis with parasellar extension $(1,2,3,4)$. This case presented increment in pituitary size that reduced after chemotherapy (Fig. 2).

The diagnosis of autoimmune hypophysitis in our patient is less likely for several reasons. First, there is no autoimmune disease background. Second, it is not the usual age of presentation. Third, the finding of atypical lymphocytes in the lumbar puncture confirming CNS infiltration, and, fourth, the normalization of pituitary gland and infundibulum's anatomical and functional characteristics after chemotherapy. On the other hand, autoimmune hypophysitis rarely causes panhypopituitarism. Also, it can be argued that the impressive normalization of infundibulum and pituitary size at MRI (Fig. 1) may be a consequence of steroids used with CHOP chemotherapy. Although hypophysitis is treated with steroids, responsiveness is usually incomplete in terms of size and hormonal function recovery. Also, steroid responsiveness takes 4 months in average, whereas in our patient, complete recovery was confirmed after one month $(10,11)$. Currently, hypophysitis treatment with high-dose steroids is no longer recommended by some authors as nearly 70\% showed steroids-related adverse reactions, and pituitary recovery is similar to that seen with clinical observation $(10,11)$. In contrast, lymphoma commonly shows important and quick size reduction of tumor few days after chemotherapy is started. Therefore, we suggest that lymphoma responsiveness explained the complete resolution of the infundibulum-hypophysitislike MRI image after one month of treatment in the case presented (Fig. 1). 
The treatment of NHL infiltration to CNS aims to substitute hormonal deficiencies. Some cases recover pituitary function after an aggressive chemotherapy treatment and bone marrow transplantation (4).

In summary, we report here an interesting and rare case of DLBCL subtype with pituitary gland and stalk infundibulum infiltration. Also, it resulted in a diagnostic challenge as both anterior and posterior pituitary functions were affected, which is uncommon. Finally, MRI was also atypical with similar characteristics of those seen in infundibulum-hypophysitis which resolved after chemotherapy treatment.

\section{Declaration of interest}

The authors declare that there is no conflict of interest that could be perceived as prejudicing the impartiality of the research reported.

\section{Funding}

This research did not receive any specific grant from any funding agency in the public, commercial or not-for-profit sector

\section{Patient consent}

Written informed consent has been obtained from the patient for the publication of the submitted article.

\section{Author contribution statement}

Each co-author was involved in the diagnosis and follow-up of the patient. L S A and D C R wrote the manuscript and did picture editing. All authors reviewed and approved the manuscript.

\section{References}

1 Kumabe A, Kenzaka T \& Nishimura Y 2015 A rare case of anasarca caused by infiltration of the pituitary gland by diffuse large B-cell lymphoma. BMC Endocrine Disorders 15 1-5. (doi:10.1186/s12902015-0007-4)

2 Valeros KA \& Khoo E 2014 Anterior panhypopituitarism in diffuse large B-cell stage IV lymphoma. Journal of Clinical Neuroscience 21 1464-1466. (doi:10.1016/j.jocn.2013.10.042)

3 Yang J, Zhao N, Zhang G \& Zheng W 2013 Clinical features of patients with non-Hodgkin's lymphoma metastasizing to the pituitary glands. Oncology Letters 5 1643-1648. (doi:10.3892/ol.2013.1266)

4 Nakashima Y, Shiratsuchi M \& Abe I 2013 Pituitary and adrenal involvement in diffuse large B-cell lymphoma, with recovery of their function after chemotherapy. BMC Endocrine Disorders 13 2-7. (doi:10.1186/1472-6823-13-2)

5 Orija IB, Weil JR \& Burkhardt RE 2012 Pituitary incidentaloma. Best Practice and Research Clinical Endocrinology and Metabolism 26 47-68. (doi:10.1016/j.beem.2011.07.003)

6 Fasset DR \& Couldwell WT 2004 Metastases to the pituitary gland. Neurosurgical Focus 16 1-4. (doi:10.3171/foc.2004.16.4.9)

7 Spinelli GP, Lo Russo G, Miele E, Prinzi N, Tomao F, Antonelli M, Giangaspero F, Stati V, Strudel M \& Tomao S 2012 Breast cancer metastatic to the pituitary gland: a case report. World Journal of Surgical Oncology 10 137. (doi:10.1186/1477-7819-10-137)

8 Giustina A, Gola M, Doga M \& Rosei EA 2001 Primary lymphoma of the pituitary: an emerging clinical entity. Journal of Clinical Endocrinology and Metabolism 86 4567-4575. (doi:10.1210/ jcem.86.10.7909)

9 Naysmith A, Hancock BW, Cullen DR, Richmond J \& Wilde CE 1976 Pituitary function in patients receiving intermittent cytotoxic and corticosteroid therapy for malignant lymphoma. Lancet 1 715-717. (doi:10.1016/S0140-6736(76)93090-7)

10 Caturegli P, Newschaffer C \& Olivi A 2005 Autoimmune hypophysitis Endocrine Reviews 26 599-614. (doi:10.1210/er.2004-0011)

11 Honegger J, Buchfelder M, Schlaffer S, Droste M, Werner S, Strasburger C, Störmann S, Schopohl J, Kacheva S, Deutschbein T, et al. 2015 Pituitary Working Group of the German Society of Endocrinology. Treatment of primary hypophysitis in Germany. Journal of Clinical Endocrinology and Metabolism 100 3460-3469. (doi:10.1210/jc.2015-2146)

Received in final form 24 November 2016

Accepted 1 December 2016 